\title{
Black hole mass measurements using ionized gas discs: systematic dust effects
}

\author{
Maarten Baes \\ Sterrenkundig Observatorium, Universiteit Gent, Krijgslaan 281 S9, B-9000 Gent, Belgium
}

\begin{abstract}
Using detailed Monte Carlo radiative transfer simulations in realistic models for galactic nuclei, we investigate the influence of interstellar dust in ionized gas discs on the rotation curves and the resulting black hole mass measurements. We find that absorption and scattering by interstellar dust leaves the shape of the rotation curves basically unaltered, but slightly decreases the central slope of the rotation curves. As a result, the "observed" black hole masses are systematically underestimated by some 10 to $20 \%$ for realistic optical depths. We therefore argue that the systematic effect of dust attenuation should be taken into account when estimating SMBH masses using ionized gas kinematics.
\end{abstract}

\section{INTRODUCTION}

Measuring the kinematics of ionized gas disks has become one the most important methods to determine the masses of supermassive black holes (SMBHs) in the nuclei of nearby galaxies. The state-of-the-art modeling techniques are refined to a high degree and take into account the major relevant astrophysical processes as well as the main instrumental effects. Applying such modeling techniques to high-quality HST data, a formal SMBH mass measurement uncertainty of $25 \%$ or better has been claimed with well-behaved disks (e.g. [4], [6], [1], [5]). Many ionized gas disks contain substantial amounts of interstellar dust. As optical $\mathrm{H} \alpha$ radiation is easily absorbed and scattered by interstellar dust grains, these effects might affect the observed rotation curve and hence the SMBH mass estimate. The goal of our investigation is to determine the importance of this effect through detailed Monte Carlo simulations.

\section{THE MODEL}

We have set up a detailed radiative transfer model to investigate the effects of neglecting dust absorption and scattering on ionized gas disc rotation curves and the corresponding SMBH masses in a typical galactic nucleus. Our model consists of a thin (but not infinitesimally thin) axisymmetric double-exponential disc rotating in a galactic nucleus. The intrinsic rotation velocity of the disc is determined by the combined gravitational potential of a stellar distribution and a central SMBH. We consider various SMBH masses, up to $M_{\bullet}=10^{8} M_{\odot}$. An additional isotropic bulk velocity dispersion is taken into account. We assume that a fraction of the disc consists of interstellar dust with typical Milky Way dust optical properties. The dust is assumed to be coupled to the ionized 

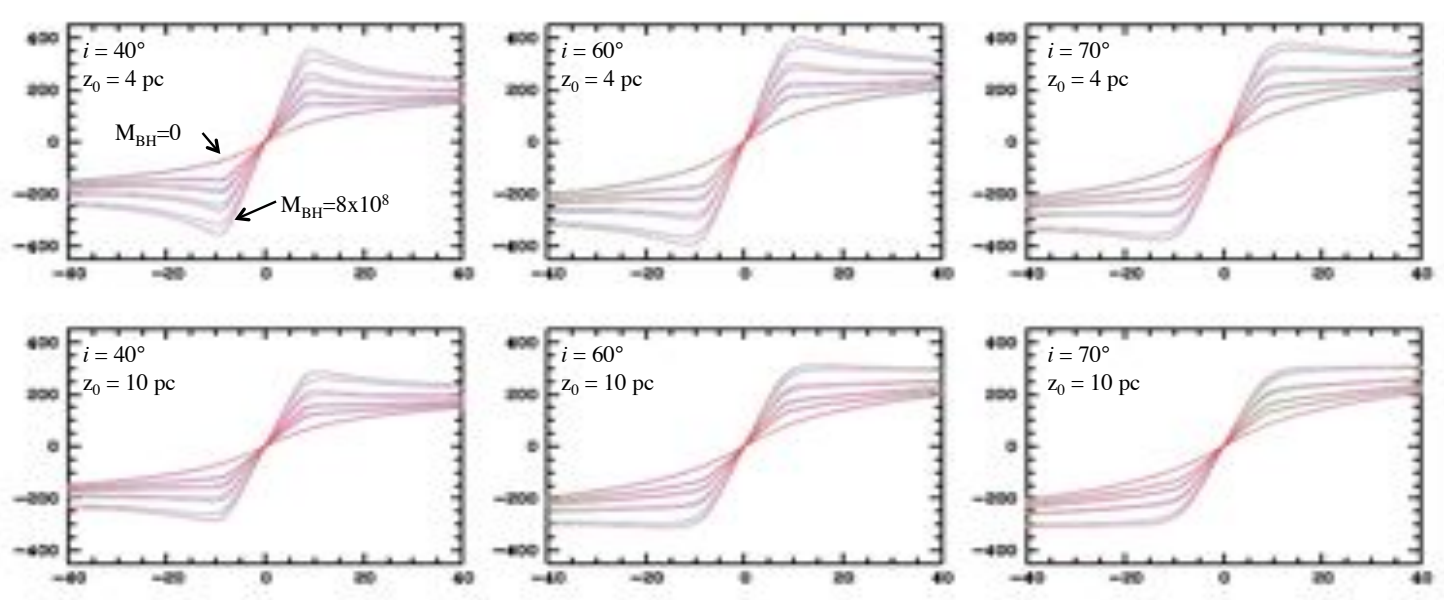

FIGURE 1. Nuclear rotation curves for dusty ionized gas discs. Each panel corresponds to a particular choice for the inclination $i$ and the disc scale height $z_{0}$. In each panel we plot the rotation curves for different black hole masses (ranging between 0 and $8 \times 10^{8} \mathrm{M}_{\odot}$ ) and for different optical depths (ranging between 0 and 2). For any $i, z_{0}$ and $M_{\bullet}$, the slope of the rotation curve slightly decreases with increasing optical depth.

gas and has exactly the same spatial and velocity distribution. The SMBH mass $M_{\bullet}$, the thickness $z_{0}$ of the disc and the V-band optical depth $\tau_{\mathrm{v}}$, a quantity equivalent to the dust mass, are free parameters in our models.

\section{SKIRT RADIATIVE TRANSFER MODELLING}

For each model, we calculate the observed line-of-sight velocity field (data cubes) at several inclination angles. We use the SKIRT code, a 3D Monte Carlo radiative transfer code that has been developed explicitly to model the kinematics of dusty galaxies [2], [3]. The code uses photon packages that carry around information on the line-of-sight velocity of the sources (in this case the $\mathrm{H} \alpha$ emitting gas) that emitted them. The effects of absorption and multiple anisotropic scattering are properly taken into account. During scattering events, the Doppler information carried by each photon package is updated, taking into account both the original line-of-sight velocity and the scattering dust grain velocity. When the photon packages leave the system, their "observed" line-of-sight velocity information can be extracted, which is used to build up data cubes. At the end of the simulation, we calculate rotation velocity maps and velocity dispersion maps from the simulated data cubes. We focus in particular on the major axis rotation curves.

\section{RESULTS}

In Figure 1 we plot a set of nuclear rotation curves for our dusty ionized gas discs. The different panels correspond to different values for $z_{0}$ and the inclination angle. Within each individual panel, rotation curves are shown for different values of $M_{\bullet}$ and $\tau_{\mathrm{v}}$. For 

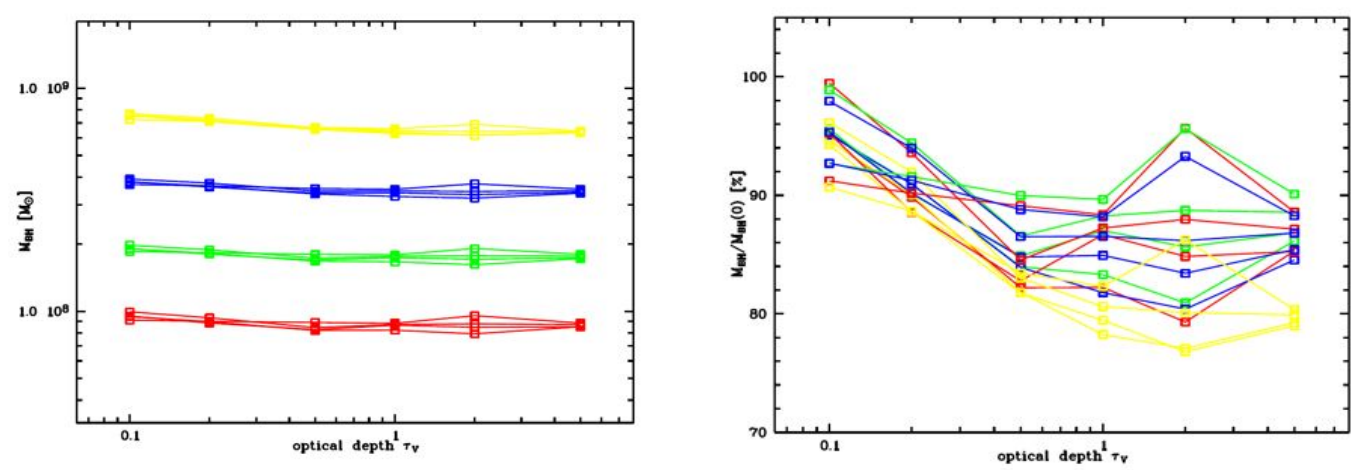

FIGURE 2. Left panel: An estimate of the "observed" black hole mass of the galaxy, derived from the observed dust-affected rotation curve, explicitly as a function of optical depth. The different colours correspond to different intrinsic SMBH masses, the different lines correspond to different inclination angles. - Right panel: The ratio between the "observed" and the intrinsic SMBH mass, explicitly as a function of optical depth. SMBH masses are systematically underestimated if dust attenuation is not properly taken into account.

a fixed spatial resolution and inclination, the shape of the rotation curve of dust-free models depends on the SMBH mass and disc thickness. In particular, increasing disc thickness has a significant influence due to the larger integration along the line of sight. Modeling ionized gas disks as infinitesimally thin disks might introduce a bias.

To investigate the effect of interstellar dust absorption and scattering, we must compare models with different values of the optical depth. The curves corresponding to various $\tau_{\mathrm{v}}$ basically coincide: apparently absorption and scattering by interstellar dust leaves the shape of the rotation curves qualitatively unaltered. However, close inspection shows that an increasing amount of interstellar dust slightly decreases the central slope of the rotation curves. We estimate the bias one makes by neglecting the effect of interstellar dust on SMBH mass estimates by translating the observed rotation curve central slopes into SMBH masses. Figure 2 shows the effect of increasing dust mass on the "observed" SMBH mass and on the ratio between the "observed" and the intrinsic SMBH mass. It clearly demonstrates that, as the optical depth increases, the observed SMBH mass decreases, i.e. the true SMBH mass is more and more underestimated. This means that neglecting interstellar dust when determining SMBH masses from ionized gas kinematics systematically leads to an underestimate of the black hole mass. For $\tau_{\mathrm{v}} \sim 0.2$ the SMBH is underestimated by about $10 \%$, for $\tau_{\mathrm{v}}>1$ the black hole mass is underestimated by 10 to $20 \%$. This effect is more or less independent of the SMBH mass and the inclination of the disc.

\section{CONCLUSIONS}

We find that absorption and scattering by interstellar dust leaves the shape of the rotation curves basically unaltered, but slightly decreases the central slope of the rotation curves. Since the central slope can be used as a proxy for the SMBH mass, this result means that "observed" black hole masses are systematically underestimated by some 10 to $20 \%$ 
for realistic optical depths. Our study demonstrates that the systematic effect of dust attenuation should be taken into account in SMBH demographics studies.

\section{REFERENCES}

1. Atkinson J. W., Collett J. L., Marconi A., et al., 2005, MNRAS, 359, 504

2. Baes M., Dejonghe H., 2002, MNRAS, 335, 441

3. Baes M., Davies J. I., Dejonghe H., et al., 2003, MNRAS, 343, 1081

4. Barth A. J., Sarzi M., Rix H.-W., et al., 2001, ApJ, 555, 685

5. Häring-Neumayer N., Cappellari M., Rix H.-W., et al., 2006, ApJ, 643, 226

6. Marconi A., Axon D. J., Capetti A., et al., 2003, ApJ, 586, 868 